\title{
Clinical features and characteristics of uveitis associated with juvenile idiopathic arthritis in Japan: first report of the pediatric rheumatology association of Japan (PRAJ)
}

Junko Yasumura $^{1 *}$ (D), Masato Yashiro ${ }^{2}$, Nami Okamoto $^{3}$, Kosuke Shabana $^{3}$, Hiroaki Umebayashi ${ }^{4}$, Naomi Iwata $^{5}$, Yuka Okura ${ }^{6}$, Tomohiro Kubota ${ }^{7}$, Masaki Shimizu ${ }^{8}$, Minako Tomiita ${ }^{9}$, Yasuo Nakagishi ${ }^{10}$, Kenichi Nishimura ${ }^{11}$, Ryoki Hara ${ }^{11}$, Mao Mizuta ${ }^{8}$, Takahiro Yasumi ${ }^{12}$, Fumiya Yamaide ${ }^{13}$, Hiroyuki Wakiguchi ${ }^{14}$, Masao Kobayashi ${ }^{1}$ and Masaaki Mori ${ }^{15}$

\section{Abstract}

Background: Although there are many reports on Juvenile Idiopathic arthritis-associated uveitis (JIA-U) from various countries, especially from Europe and North America, there are few reports from Asia. Our aim was to investigate the epidemiology, characteristics and predictors of JIA-U in Japan.

Methods: Data were retrospectively collected on 726 patients with JIA from medical records as of April 2016 at 15 medical centers specialized in pediatric rheumatic diseases. Of these, patients with uveitis were further investigated for the specific characteristics of this manifestation.

Results: The prevalence of uveitis was $6.1 \%$ in the $726 \mathrm{JIA}$ patients examined. Incidence of uveitis was significantly higher in patients with an earlier arthritis onset (2.6-vs.-5.8 years, $P<0.0001)$, oligoarthritis $(16.1 \%$-vs.-1.6\%, $P<0.001)$, or anti-nuclear antibodies. On the contrary, it was significantly less common in patients with rheumatoid factor or anti-cyclic citrullinated peptide antibodies. A history of using methotrexate (MTX), infliximab or adalimumab was also associated with uveitis occurrence. The median age at uveitis diagnosis was 5 years, and the median time from arthritis onset to uveitis diagnosis was 2 years. The occurrence of anterior and bilateral uveitis was 79.3 and $53.7 \%$, respectively. There were no symptoms at uveitis diagnosis in $58.5 \%$ of cases. Complications arising between the time of uveitis diagnosis and the last observation increased from 31.7 to 56.1\%; in particular, cataract was increased 3 -fold. While no patients lost their vision, $61.9 \%$ did not recover normal vision ( $\geq 1.0)$, and in many cases active uveitis persisted, especially in males. In addition to steroid eye drops (97.6\%) and MTX (15.4\%), biological agents were used for treating the uveitis in $41.5 \%$ of patients.

Conclusions: The epidemiology, characteristics and predictors of JIA-U in Japan are described here for the first time. Although the prevalence of JIA-U in Japan is lower than in predominantly Caucasian cohorts, as reported from North America and Europe, the epidemiology, characteristics and predictors were found to be similar.

Keywords: Juvenile idiopathic arthritis, Uveitis, Epidemiology, Asian

\footnotetext{
* Correspondence: junko-ma562@hiroshima-u.ac.jp

${ }^{1}$ Department of Pediatrics, Hiroshima University Graduate School of

Biomedical and Health Sciences, 1-2-3 Kasumi, Minami-ku, Hiroshima

734-8551, Japan

Full list of author information is available at the end of the article
}

(c) The Author(s). 2019 Open Access This article is distributed under the terms of the Creative Commons Attribution 4.0 International License (http://creativecommons.org/licenses/by/4.0/), which permits unrestricted use, distribution, and reproduction in any medium, provided you give appropriate credit to the original author(s) and the source, provide a link to the Creative Commons license, and indicate if changes were made. The Creative Commons Public Domain Dedication waiver (http://creativecommons.org/publicdomain/zero/1.0/) applies to the data made available in this article, unless otherwise stated. 


\section{Background}

Uveitis is the most common extra-articular manifestation of juvenile idiopathic arthritis (JIA); it is a serious manifestation carrying the risk of blindness if treatment is delayed or inadequate. The prevalence of JIA-associated uveitis (JIA-U) has been reported as ranging from 4.7 to $20.5 \%$ [1-6] with local differences noted. Numerous reports have identified risk factors for JIA-U such as female sex, oligoarthritis, earlier arthritis onset, ANA-positivity, and RF-negativity in predominantly Caucasian cohorts $[2,7,8]$. Do these characteristics also apply to JIA-U in East Asia? To date, there are very few reports from East Asia and the epidemiology, characteristics and risk factors for JIA-U in Japan are unclear.

Although we, the members of the Pediatric Rheumatology Association of Japan (PRAJ), issued recommendations for ophthalmologic screening intervals for JIA patients in Japan $[9,10]$ (Table 1), these were based on other countries' guidelines $[2,11]$. In any event, these recommendations were issued only one year before this study of patients in Japan, and therefore such recommendations did not apply to most of the patients included here. Given that the prevalence and characteristics of JIA-U in Japan were unclear, and pediatricians and ophthalmologists had little knowledge of the management of JIA- $\mathrm{U}$, investigating these issues specifically in Japanese patients was warranted. Accordingly, in the present study, we aimed to establish the epidemiology, clinical characteristics, and risk factors for JIA-U in Japan and to compare these with data reported from other countries. Thus, we reviewed the charts of outpatients making regular hospital visits as of April 2016.

\section{Methods}

\section{Study setting}

This is a retrospective study, approved by the Ethics Review Board of Tokyo Medical and Dental University
(No. M2015-537), the main study center, on March 4th, 2016, after which approval was obtained from each of the 15 other participating medical centers. Guardians and patients were provided information by means of an opt-out form.

\section{Study design and patients}

First, we sent questionnaires to pediatricians who are members of the PRAJ belonging to 15 medical centers to gather data on the characteristics of JIA in Japan. We analyzed data from outpatients with JIA who had regularly visited the hospital as of April 2016. All patients were classified according to the International League of Associations for Rheumatology (ILAR) criteria [12]. Second, we undertook a further questionnaire survey for patients identified as having uveitis in the first questionnaire, and gathered more detailed information about uveitis features from their ophthalmology charts using this second questionnaire. Patients who had already discontinued follow-up of JIA as of April 2016 were not included.

\section{Data collection}

All patient data including ophthalmic records were collected from medical histories and were recorded by pediatricians. The following parameters were evaluated for the primary investigation: sex, age as of April 2016, duration of disease, age at arthritis diagnosis, type of JIA classified by ILAR criteria, laboratory data including anti-nuclear antibody (ANA), rheumatoid factor (RF), anti-cyclic citrullinated peptide (CCP) antibody, serum matrix metalloproteinase-3 (MMP-3), and drugs used for treatment. An ANA titer of $\geq 1: 160$ by fluorescent antibody testing was designated positive. Data on ANA, RF and MMP-3 were acquired at the time of arthritis diagnosis. The following parameters were evaluated in

Table 1 Recommendations for ophthalmologic screening intervals for JIA patients in Japan [9, 10]

\begin{tabular}{|c|c|c|c|}
\hline \multirow[t]{2}{*}{ JIA category } & \multirow{2}{*}{$\begin{array}{l}\text { ANA } \\
\text { (titer) }^{a}\end{array}$} & \multicolumn{2}{|c|}{ Ophthalmologic screening intervals } \\
\hline & & Onset age of $\leq 6$ years old & Onset age $\geq 7$ years old \\
\hline \multicolumn{4}{|l|}{$\leq 4$ years from onset of arthritis } \\
\hline \multirow[t]{2}{*}{ Oligoarthrits, RF-negative polyarthritis, undifferentiated arthritis } & $\geq 160$ & Every 3 months & Every 6 months \\
\hline & $<160$ & Every 6 months & Every 6 months \\
\hline Psoriatic arthritis whose onset age is $<4$ years old & Regardless & Every 3 months & - \\
\hline Others & Regardless & Every 12 months & Every 12 months \\
\hline \multicolumn{4}{|l|}{$4<$ years, $\leq 7$ years from onset of arthritis } \\
\hline \multirow[t]{2}{*}{ Oligoarthrits, RF-negative polyarthritis, undifferentiated arthritis } & $\geq 160$ & Every 6 months & Every 12 months \\
\hline & $<160$ & Every 12 months & Every 12 months \\
\hline Psoriatic arthritis whose onset age is $<4$ years old & Regardless & Every 6 months & - \\
\hline Others & Regardless & Every 12 months & Every 12 months \\
\hline$>7$ years from onset of arthritis: Every 12 months. & & & \\
\hline
\end{tabular}


the second investigation: race of parents, family history of uveitis, infection history, possession of HLA-B27, location of uveitis, age at uveitis diagnosis, time from arthritis onset to uveitis diagnosis, timing of uveitis diagnosis, eye manifestations at uveitis diagnosis, eye complications due to uveitis at the first and last observation, activity of arthritis at uveitis diagnosis, current activity of arthritis, current activity of uveitis, visual acuity at uveitis diagnosis and at present, drugs used for treatment. Visual acuity was recorded as a decimal unit using the Landolt ring method where 1.0 refers to $20 / 20$ of the Snellen fraction, whereas 0.1 refers to 20/200 [13].

\section{Statistical analysis}

Statistical analysis was performed using JMP pro $^{\circ}$ Version 13 software (SAS Institute Inc., Cary, NC, USA). The Wilcoxon rank sum test or Welch's $t$ test were used for comparison of continuous variables between two groups, and categorical variables were analyzed using the Chi-Square test or Fisher's exact test (two-sided). Odds ratios (ORs) are presented with $95 \%$ confidence intervals $(\mathrm{CI})$. The time from onset of arthritis to diagnosis of uveitis was analyzed by the Kaplan-Meier method. A $p$-value $<0.05$ was considered significant.

\section{Results}

\section{Comparison of characteristics of JIA patients with and without uveitis}

We collected data from 730 patients with JIA at 15 medical centers. Four patients were excluded due to many missing data. Of the remaining 726 JIA patients, 44 (6.1\%) had uveitis. Table 2 shows the characteristics of patients with and without uveitis. There were no significant differences between the two groups in sex or age at the last visit. Patients with uveitis had been significantly younger at arthritis onset (2.6-vs.-5.8 years, $P<0.0001)$ and the duration of disease was significantly longer (9.1-vs.-5.1 years, $P<0.0001)$ than in patients without uveitis. The most frequent disease type in patients with uveitis was oligoarthritis (81.8\%), followed by RF-negative polyarthritis, but there were no patients with systemic arthritis or RF-positive polyarthritis. There were very few patients with psoriatic arthritis or non-classified arthritis in either group. The rate of uveitis in oligoarthritis patients was $16.1 \%$ and in all other JIA patients 1.6\%; thus, the risk of uveitis in oligoarthritis was 10 -fold higher (Table 6). In addition, the frequency of ANA-positive patients was significantly higher in the group with uveitis $(52.3 \%$-vs.-22.2\%, $P<0.0001)$, with a tendency towards higher ANA titers than patients without uveitis (ANA titers $\geq 1: 640 ; P=0.018$ ) (data not shown). The frequency of RF-positive cases (2.4\%-vs.-24.5\%, $P=0.0004)$ and those with anti-CCP antibodies $(0 \%$-vs.-26.2\%, $P<0.0001)$ was significantly lower in the uveitis group. No significant differences in serum MMP-3 levels at JIA diagnosis were noted between the two groups. The proportion of patients with a history of using oral Methotrexate (MTX) was significantly higher in patients with uveitis (95.5\%-vs.-77.0\%, $P$ $=0.0022$ ). Similarly, significant differences were noted for infliximab (IFX), adalimumab (ADA), and tocilizumab (TCZ) use: IFX and ADA were more frequently used in the uveitis group, whereas TCZ was more frequently used by patients without uveitis.

\section{Clinical characteristics of patients with uveitis}

We obtained data for a further analysis of 41 of the 44 patients with uveitis. Three patients were excluded due to lack of data from their physicians. Of the 41 patients, 38 had Japanese parents, one had Chinese parents, one had a Chinese and a Japanese parent, and the other had a Chinese and an Iranian parent. They were found to have no family history of uveitis or history of preceding infections. HLA-B27 had been tested only in $34.1 \%$ (14 of 41 patients) because of not being covered by insurance. Two patients were positive, and 12 patients were negative.

We were able to obtain data on the site of uveitis from only 29 of the 41 patients because of poor record keeping by ophthalmologists in some centers. Uveitis in 29 patients was anterior in $79.3 \%(N=23)$, panuveitis in $13.8 \%(N=4)$, posterior in $3.5 \%(N=1)$, and both anterior and posterior in $3.5 \%(\mathrm{~N}=1)$. Uveitis was bilateral in $53.7 \%$ of these 41 patients.

The median age at uveitis diagnosis was 5 years (interquartile ranges: 25th-75th percentile, 3-7); uveitis occurred before the 5th and 8th birthday in 43.9 and 80.5\% of patients, respectively. Median time from arthritis onset to uveitis diagnosis was 2 years (interquartile ranges: 25th-75th percentile, 1-5). Time from arthritis onset to uveitis diagnosis is shown Fig. 1, including two patients in which uveitis was diagnosed even before the arthritis. Time from arthritis onset to uveitis diagnosis was $<4$ years in $63.4 \%$ and $<7$ years in $95.1 \%$ of patients. The timing of uveitis diagnosis was "prior to arthritis onset" in $4.9 \%$, "at the same time as arthritis diagnosis" in $36.6 \%$, "during on medication" in $36.6 \%$, and "during off medication remission" in $19.5 \%$ of the patients. Of the 15 (36.6\%) patients who developed uveitis during arthritis treatment, 13 were being treated with oral MTX, one with non-steroidal anti-inflammatory drugs $(\mathrm{N}$ SAIDs), and the remaining patient with NSAIDs plus glucocorticoids (GC). One patient used a biological agent, TCZ. Uveitis developed in 9 patients from 4 months to 6 years after discontinuation of arthritis treatment; for 8 of the 9 it was within 3 years. While $56.1 \%$ had arthritis at uveitis diagnosis, $41.5 \%$ of patients 
Table 2 Comparison of characteristics in 682 non-uveitis patients vs. 44 JIA-U patients

\begin{tabular}{|c|c|c|c|c|}
\hline & JIA total & without uveitis & with uveitis & $P-$ value $^{c}$ \\
\hline Number of Patients, N (\%) & 726 & $682(93.9 \%)$ & $44(6.1 \%)$ & \\
\hline \multicolumn{5}{|l|}{ Variables } \\
\hline Female, N (\%) & $492(67.8 \%)$ & $461(67.6 \%)$ & $31(70.5 \%)$ & 0.743 \\
\hline Age at the last visit, $\mathrm{N}$ & 724 & 680 & 44 & \\
\hline median ${ }^{a}$ (yrs.) & $12.9(8.9-17.2)$ & $12.8(8.8-17.2)$ & $13.6(10.1-18.3)$ & 0.191 \\
\hline Duration of disease at the last visit, $\mathrm{N}$ & 718 & 674 & 44 & \\
\hline median ${ }^{a}$ (yrs.) & $5.4(2.8-9.2)$ & $5.1(2.7-8.8)$ & $9.1(6.5-14.3)$ & $<0.0001^{*}$ \\
\hline Age at arthritis onset, $\mathrm{N}$ & 720 & 676 & 44 & \\
\hline median ${ }^{a}$ (yrs.) & $5.5(2.7-10.3)$ & $5.8(2.8-10.4)$ & $2.6(1.6-5.1)$ & $<0.0001^{*}$ \\
\hline \multicolumn{5}{|l|}{ Subtypes of JIA } \\
\hline Oligo persistent, N (\%) & $184(25.3 \%)$ & $155(22.7 \%)$ & $29(65.9 \%)$ & $<0.001^{*}$ \\
\hline Oligo extended & $40(5.5 \%)$ & $33(4.8 \%)$ & $7(15.9 \%)$ & $0.0076^{*}$ \\
\hline Poly RF (-) & $95(13.1 \%)$ & $90(13.2 \%)$ & $5(11.4 \%)$ & 1.000 \\
\hline Poly RF (+) & $152(20.9 \%)$ & $152(22.3 \%)$ & $0(0 \%)$ & $<0.001^{*}$ \\
\hline Systemic & 204 (28.1\%) & 204 (29.9\%) & $0(0 \%)$ & $<0.001^{*}$ \\
\hline Psoriatic & $4(0.6 \%)$ & $4(0.6 \%)$ & $0(0 \%)$ & 1.000 \\
\hline Enthesitis related & $37(5.1 \%)$ & $35(5.1 \%)$ & $2(4.5 \%)$ & 1.000 \\
\hline Undifferentiated & $10(1.4 \%)$ & $9(1.3 \%)$ & $1(2.3 \%)$ & 0.467 \\
\hline \multicolumn{5}{|l|}{ Blood test } \\
\hline ANA tested & 615 & 571 & 44 & \\
\hline Positive $^{b}, \mathrm{~N}(\%)$ & $150(24.4 \%)$ & $127(22.2 \%)$ & $23(52.3 \%)$ & $<0.0001^{*}$ \\
\hline RF tested & 592 & 551 & 41 & \\
\hline Positive, N (\%) & $136(23.0 \%)$ & $135(24.5 \%)$ & $1(2.4 \%)$ & $0.0004^{*}$ \\
\hline Anti-CCP antibody tested & 445 & 404 & 41 & \\
\hline Positive, N (\%) & $106(23.8 \%)$ & $106(26.2 \%)$ & $0(0 \%)$ & $<0.0001^{*}$ \\
\hline MMP-3 tested & 552 & 521 & 31 & \\
\hline MMP-3 (ng/ml), mean \pm SD & $169.0 \pm 301.3$ & $170.6 \pm 308.7$ & $142.0 \pm 123.4$ & 0.27 \\
\hline \multicolumn{5}{|l|}{ Medication Use } \\
\hline Methotrexate, N (\%) & $567(78.1 \%)$ & $525(77.0 \%)$ & 42 (95.5\%) & $0.0022^{*}$ \\
\hline Infliximab & $30(4.1 \%)$ & $18(2.6 \%)$ & $12(27.3 \%)$ & $<0.0001^{*}$ \\
\hline Etanercept & $81(11.2 \%)$ & $78(11.4 \%)$ & $3(6.8 \%)$ & 0.4625 \\
\hline Adalimumab & $124(17.1 \%)$ & $109(16.0 \%)$ & $15(34.1 \%)$ & $0.0058^{*}$ \\
\hline Tocilizumab & $297(40.9 \%)$ & $289(42.4 \%)$ & 8 (18.2\%) & $0.0014^{*}$ \\
\hline Abatacept & 24 (3.3\%) & 24 (3.5\%) & $0(0 \%)$ & $0.3904^{*}$ \\
\hline
\end{tabular}

interquartile ranges: 25 th -75 th percentile, ${ }^{b}$ fluorescent ANA $\geq 1: 160$, 'Statistically significant at $5 \%$ level of significance; ${ }^{*} p=<0.05$

Chi-square tests, Fisher's extract test (two-tailed test), Wilcoxon rank sum test

exhibited only persistent uveitis at the final observation, and not arthritis.

Ocular symptoms were present at uveitis diagnosis in $36.6 \%$ of patients, whereby ocular hyperemia accounted for $46.7 \%$ and decreased vision accounted for $46.7 \%$ (Table 3). Ocular complications were noted in $31.7 \%$ of patients at diagnosis (three were unknown) and in 56.1\% at the final observation. The major complications at diagnosis were posterior synechia of the iris (46.2\%), followed by cataract (30.8\%), band-keratopathy (30.8\%), and glaucoma (23.1\%). At the time of the last observation, the number of patients with cataract had increased 3 -fold since uveitis diagnosis (Table 4). There were no significant differences in oral GC use between patients with or without cataracts at the final observation. There were also no significant differences between patients with or without complications at diagnosis in the proportions of patients with active uveitis at the final 


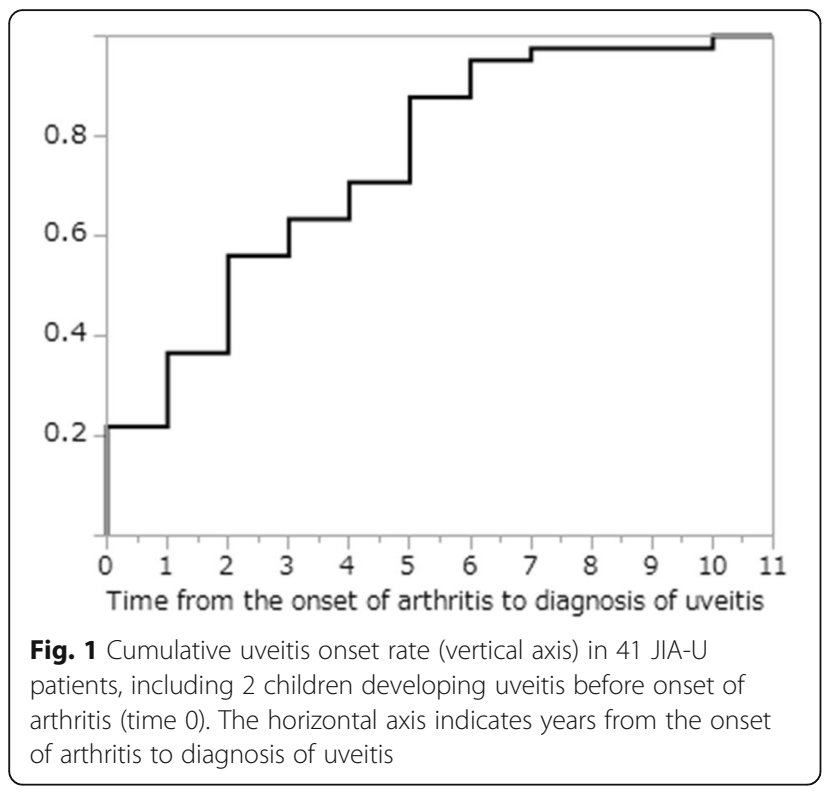

observation ( $P=0.09$, OR: 3.6, 95\% CI: 0.9-14.4). However, patients with complications at diagnosis had significantly more complications at the final observation $(P=$ 0.014, OR: 5.57, 95\% CI: 1.51-20.51). No significant differences were noted between the presence or absence of complications at uveitis diagnosis and the age of the patients at the time of uveitis diagnosis, or time from arthritis onset to uveitis diagnosis.

We obtained visual acuity data on 63 affected eyes (30 right eyes and 33 left eyes) in 39 patients. Two were excluded because of lack of data of the side of uveitis and visual acuity. We did not identify any patient who lost vision during the observation period. However, visual acuity of the affected eye less than 0.1 was noted in $6.7 \%$ at diagnosis (4 of 60 eyes: 3 data points were missing), and in $6.4 \%$ at the final observation ( 4 of 63 eyes). Visual acuity less than 1.0 at the final observation was noted in $61.9 \%$ (39 of 63 eyes) and less than 0.5 was noted in $22.2 \%$ (14 of 63 eyes). No significant differences were seen between complications at diagnosis and at the final observation, and prognosis of visual capacity.

Table 3 Ocular symptoms at uveitis diagnosis $\left(N=41^{\mathrm{a}}\right)$

\begin{tabular}{ll}
\hline & $\mathrm{N}(\%)$ \\
\hline Ocular symptom (+) & $15(36.6 \%)$ \\
Decreased vision & $7(46.7 \%)$ \\
Ocular hyperemia & $7(46.7 \%)$ \\
Photophobia & $2(13.3 \%)$ \\
Ocular pain & $1(6.7 \%)$ \\
Blurred vision & $1(6.7 \%)$ \\
Myodesopsia (floaters) & $1(6.7 \%)$ \\
White pupil & $1(6.7 \%)$ \\
\hline
\end{tabular}

${ }^{a} 2$ data were unknown. There were patients who had multiple symptoms
Table 4 Ocular complications in JIA-U patients ${ }^{\mathrm{a}}$

\begin{tabular}{lll}
\hline & $\begin{array}{l}\text { at uveitis diagnosis } \\
\left(N=41^{b}\right) \\
N(\%)\end{array}$ & $\begin{array}{l}\text { at the last visit } \\
(N=41) \\
N(\%)\end{array}$ \\
\hline Ocular complication (+) & $13(31.7 \%)$ & $23(56.1 \%)$ \\
Cataract & $4(30.8 \%)$ & $12(52.2 \%)$ \\
Glaucoma & $3(23.1 \%)$ & $5(21.7 \%)$ \\
Posterior synechia & $6(46.2 \%)$ & $4(17.4 \%)$ \\
Band-keratopathy & $4(30.8 \%)$ & $7(30.4 \%)$ \\
Other complications & $1(7.7 \%)^{c}$ & $2(8.7 \%)^{d}$
\end{tabular}

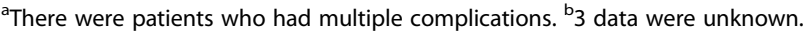
cpapilledema. ${ }^{\mathrm{d}}$ divergent strabismus and Iridocyclitis scar

\section{Uveitis treatment}

Steroid eye drops were mainly used for uveitis treatment (97.6\%). Only two patients received steroid injections into the eye. MTX was used in $34.1 \%$ (14/41 patients) at diagnosis of uveitis, and in 95.1\% (39/41 patients) after diagnosis. Physicians used MTX for treating the uveitis in only $15.4 \%$ (6/39 patients) after diagnosis of uveitis. On the other hand, biological agents were used in $70.7 \%$ of patients and for treating uveitis in $41.5 \%$. IFX (29.3\%) and ADA (36.6\%) were the most frequently-used agents. A history of some type of surgery was noted in $36.6 \%$ of the patients. Of these, $86.7 \%$ (13/15 patients) underwent cataract surgery, most of which were phacoemulsification and intraocular lens implantation. There was no significant association between the presence of cataract at the time of final observation and a history of surgery $(P$ $=0.0834)$. Other surgery included vitrectomy, trabeculectomy, perioperative surgery and phototherapeutic keratectomy.

\section{Sex differences}

Risk factors were compared between males and females in the group with uveitis (Table 5). No significant sex differences were seen for age at uveitis diagnosis. Although there was also no significant difference in age at onset of arthritis in either males or females without uveitis (6.9 \pm 4.4 -vs. $-6.6 \pm 4.3$ years, $P=0.37$, data not shown), females in the uveitis group had a significantly earlier arthritis onset $(2.3 \pm 1.9$ vs. $4.5 \pm 3.0$ years old, $P$ $=0.032$ ). While the percentage of ANA-positive females at diagnosis of JIA was significantly higher in patients without uveitis $(27.8 \%$-vs.- $10.1 \%, P<0.0001$, data not shown), there were no significant sex differences in the uveitis group. There was a tendency for more female than male patients to have anterior uveitis at diagnosis, but this difference was not significant (94.1\%-vs.-58.3\%, $P=0.0563)$. The proportion of patients without ocular symptoms at diagnosis was higher in females than males (74.1\%-vs.-33.3\%, $P=0.031$ ). Although there was no significant sex difference in the proportion of patients with 
Table 5 Sex differences of characteristics in JIA-U patients

\begin{tabular}{|c|c|c|c|c|c|c|}
\hline Variables & $\mathrm{N}$ & Male & $\mathrm{N}$ & Female & $P$ & OR $(95 \% \mathrm{Cl})$ \\
\hline mean age as of April 2016 & 13 & $11.5 \pm 4.7$ & 28 & $13.8 \pm 6.4$ & 0.3737 & \\
\hline mean age at uveitis diagnosis & 13 & $6.5 \pm 3.0$ & 28 & $5.2 \pm 2.8$ & 0.2207 & \\
\hline mean age at arthritis onset & 13 & $4.5 \pm 3.0$ & 28 & $2.3 \pm 1.9$ & 0.0324 & \\
\hline mean serum MMP-3 $(\mathrm{ng} / \mathrm{ml})$ at JIA diagnosis & 11 & $145.9 \pm 150.8$ & 20 & $139.8 \pm 109.8$ & 0.69 & \\
\hline ANA $\geq 1: 160$ & 13 & $5(38.5 \%)$ & 31 & $17(54.8 \%)$ & 0.5098 & $0.51(0.14 \sim 1.93)$ \\
\hline bilateral uveitis at uveitis diagnosis & 13 & $6(46.2 \%)$ & 26 & $15(57.7 \%)$ & 0.52 & $0.63(0.16 \sim 2.4)$ \\
\hline anterior uveitis at uveitis diagnosis & 12 & $7(58.3 \%)$ & 17 & $16(94.1 \%)$ & 0.0563 & $0.09(0.01 \sim 0.89)$ \\
\hline without eye symptoms at uveitis diagnosis & 12 & $4(33.3 \%)$ & 27 & $20(74.1 \%)$ & 0.0306 & $5.71(1.3 \sim 25.0)$ \\
\hline with complications at uveitis diagnosis & 12 & $6(50.0 \%)$ & 26 & $8(30.8 \%)$ & 0.296 & $2.25(0.55 \sim 9.17)$ \\
\hline with complications at the last visit & 13 & $9(69.2 \%)$ & 28 & $13(46.4 \%)$ & 0.2 & $2.6(0.65 \sim 10.5)$ \\
\hline with persistent uveitis activity at the last visit & 13 & $10(76.9 \%)$ & 28 & $8(28.6 \%)$ & 0.0063 & $8.33(1.8 \sim 38.4)$ \\
\hline
\end{tabular}

Chi-square tests, Fisher's extract test (two-tailed test), Welch's t test

any type of complication, the proportion of males with persistent active uveitis at the final observation was significantly higher than in females $(76.9 \%$-vs.-28.6\%, $P=$ $0.006)$. In addition, there were no significant sex differences in visual acuity or decreased visual acuity at diagnosis or at final observation. Furthermore, no significant sex difference was observed in time from arthritis onset to uveitis diagnosis.

\section{Discussion}

This is the first report surveying the epidemiology and characteristics of JIA-U in Japan. Our results are compared with previous reports in Table 6. It is apparent that the prevalence of JIA-U in Japan is lower than reported earlier from North America and Europe. These previous reports revealed regional differences in the prevalence of JIA-U: 11.6-20.5\% in North America and Europe [1-5] and 4.7\% in Taiwan [6]. Another study reported that the prevalence of JIA-U in Asia is lower than in Scandinavia as well as the United States [14]. Although these differences may be due to differences in study design, there may well also be an influence of race. Because almost all of the patients assessed in our survey were Asians, this population may have a lower incidence than predominantly Caucasian populations.

Our study confirmed that oligoarthritis, earlier arthritis onset, ANA-positivity, RF-negativity and anti-CCP antibody-negativity could be risk factors for JIA-U in Japanese as well as in predominantly Caucasian populations. Several reports showed that oligoarthritis was the most common subtype of JIA with uveitis $[2,15,16]$. Likewise, in our study most patients (81.8\%) with uveitis had oligoarthritis. While oligoarthritis accounts for approximately $50 \%$ of all JIA patients in North America and Europe [2, 4, 5, 17], the proportion is smaller in Japan and many patients have systemic and RF-positive polyarthritis. The reason for the low prevalence of JIA-U in Japan may be related to the lower proportion of oligoarthritis in Japan than in North America and Europe. We compared the rate of uveitis in oligoarthritis patients and other arthritis patients between Japan and other countries (Table 6). The risk of uveitis in oligoarthritis patients in Japan is 10-fold, which is much higher than in oligoarthiritis patients in other countries. None of the patients with systemic arthritis and RF-positive polyarthritis developed uveitis - consistent with previous reports $[2,4,5,7,18]$. Because patients with these types of arthritis have little risk of developing uveitis, in such cases, autoinflammatory diseases such as Blau syndrome, or infections should be considered rather than JIA. In the present study, it was difficult to determine the frequency of the HLA-B27 allele because too few patients were tested, but Japanese JIA is characterized by a low incidence of enthesitis-related arthritis, as shown in Table 2. In addition, psoriatic arthritis is also very rare. Previous reports $[19,20]$ indicated that mean ANA titers tend to be high, with a significantly higher prevalence of ANA $\geq 1: 320$ in JIA patients with uveitis; our study also indicated a significant difference at $\geq 1: 640$.

The mean age of onset of JIA- $\mathrm{U}$ has been variably reported to be between $3.2-10.9$ years $[1,2,5,18]$; almost all cases are diagnosed before arthritis onset or within 4 years of onset $[2,4,5,15]$, and particularly within one year of the first ophthalmologic examination $[2,5]$ (not shown in Table 6). Furthermore, $10-24 \%$ patients develop uveitis before arthritis onset $[1,2,18]$. More than $80 \%$ of the locations affected by uveitis are anterior and 60.6 to $72 \%$ are bilateral $[1,2,18]$. Ocular complications including glaucoma, cataract, band-keratopathy, and posterior synechia at uveitis diagnosis were seen in between 37.3 and $56 \%$ of uveitis patients [1, 2]. Because the presence of complications will influence the visual prognosis, early diagnosis and treatment of uveitis is crucial. However, failure to have regular ocular 
Table 6 Comparison of epidemiology and characteristics of JIA-U in our results vs. in previous reports

\begin{tabular}{|c|c|c|c|c|c|}
\hline study & our study & $\begin{array}{l}\text { Saurenmann, } \\
2007[1]\end{array}$ & $\begin{array}{l}\text { Heiligenhaus, } \\
2007[2]\end{array}$ & Nordal, 2017 [5] & Angeles-Han, 2015 [18] \\
\hline country & Japan & Switzerland & Germany & Norway & $\begin{array}{l}\text { United States of } \\
\text { America }\end{array}$ \\
\hline prevalence & $6.1 \%$ & $13.1 \%$ & $12 \%$ & $20.5 \%$ & $18 \%$ \\
\hline \multicolumn{6}{|l|}{ JIA subtype } \\
\hline oligo & $81.8 \%$ & $48 \%$ & $41 \%$ & $46.1 \%$ & $78.9 \%$ \\
\hline RF + poly & $0 \%$ & $0 \%$ & $0 \%$ & $0 \%$ & $0 \%$ \\
\hline Systemic & $0 \%$ & $0.6 \%$ & $0 \%$ & $0 \%$ & $0 \%$ \\
\hline Rate of uveitis in oligo & $16.1 \%$ & $20.9 \%$ & $17.6 \%$ & $19.6 \%$ & $30.8 \%$ \\
\hline Rate of uveitis in all other type & $1.6 \%$ & $8.3 \%$ & $6.7 \%$ & $21.3 \%$ & $7.1 \%$ \\
\hline Risk ratio of uveitis in oligo & 10 & 2.5 & 2.6 & 0.9 & 4.3 \\
\hline female ${ }^{a}$ & $\begin{array}{l}67.6 \% \text { vs. } 70.5 \% \\
p=0.743\end{array}$ & $\begin{array}{l}79.6 \% \text { vs. } 63.7 \% \\
p=0.0009^{b}\end{array}$ & $\begin{array}{l}74 \% \text { vs. } 62 \% \\
p=0.012\end{array}$ & $65.6 \%$ vs. $66.3 \%$ & $\begin{array}{l}76.9 \% \text { vs. } 70.2 \% \\
p=0.332\end{array}$ \\
\hline ANA-positive (\%) ${ }^{a}$ & $\begin{array}{l}52.3 \% \text { vs. } 22.2 \% \\
p<0.0001\end{array}$ & $\begin{array}{l}80.9 \% \text { vs. } 51 \% \\
p<0.0001\end{array}$ & $\begin{array}{l}86 \% \text { vs. } 42 \% \\
p<0.01\end{array}$ & $42.5 \%$ vs. $23.2 \%$ & $\begin{array}{l}54.9 \% \text { vs. } 35.9 \% \\
p=0.017\end{array}$ \\
\hline RF-positive (\%) ${ }^{\mathrm{a}}$ & $\begin{array}{l}2.4 \% \text { vs. } 24.5 \% \\
p=0.0004\end{array}$ & - & - & $1.1 \%$ vs. $2.7 \%$ & $\begin{array}{l}0 \% \text { vs. } 11.1 \% \\
p=0.013\end{array}$ \\
\hline $\begin{array}{l}\text { the mean age of uveitis } \\
\text { onset (yrs.) }\end{array}$ & 5.6 & 6.2 & 5.2 & $\begin{array}{l}10.8 \text { (acute) and } \\
3.2 \text { (chronic) }\end{array}$ & median 4.8 \\
\hline $\begin{array}{l}\text { the mean age of arthritis } \\
\text { onset (yrs.) })^{\mathrm{a}}\end{array}$ & 3.8 vs. 6.8 & 4.3 vs. 7.3 & 3.8 vs. 7.0 & - & median 2.8 vs. 7.7 \\
\hline $\begin{array}{l}\text { time from arthritis onset to uveitis } \\
\text { diagnosis }\end{array}$ & median 2 yrs. & mean 1.8 yrs. & median 5.5 months & - & - \\
\hline $\begin{array}{l}\text { uveitis diagnosis before the arthritis } \\
\text { diagnosis }\end{array}$ & $4.9 \%$ & $12.7 \%$ & $10 \%$ & - & $24 \%$ \\
\hline anterior uveitis & $79.3 \%$ & $100 \%$ & $83 \%$ & - & $80 \%$ \\
\hline bilateral uveitis & $53.7 \%$ & $60.6 \%$ & $70 \%$ & - & $72 \%$ \\
\hline asymptomatic uveitis & $58.5 \%$ & $69.7 \%$ & - & - & - \\
\hline
\end{tabular}

${ }^{\mathrm{a} C o m p a r i s o n}$ of patients with uveitis vs. without uveitis, $p$-value. ${ }^{\mathrm{b}}$ Only girls with oligoarthritis have a risk of uveitis

examinations may lead to delayed diagnosis because JIA-U is asymptomatic and insidious in many cases [2]. In the present study, $36.6 \%$ of patients had ocular symptom at uveitis diagnosis; one of the reasons for the large number of patients with ocular symptoms may be the lack of regular routine ophthalmologic examinations in Japan. Similar to previous reports, the most commonly affected location of the uveitis was anterior, whereas fewer cases were bilateral. In our study, the median age at diagnosis of uveitis was 5 years, $80.5 \%$ of the patients developed uveitis before they reached 8 years of age, and 95.1\% developed it under 7 years of arthritis onset. Thus, the risk of onset of uveitis is high for patients under 8 years of age and under 7 years from arthritis onset. These results suggest that recommendations for standard ophthalmological follow-up of Japanese JIA patients should consider patient age, and time from arthritis onset.

Except for two patients, all were treated with MTX, a medication previously shown to be effective for uveitis
[21-23]. However, we could not determine whether treatment with MTX was effective in the present study.

Heiligenhaus et al. stated that ocular complications at the first visit to an ophthalmologist could be predictive of ocular complications at final observation [2]. In addition, Woreta et al. concluded that a short interval between arthritis and uveitis onset, ANA-positivity and the degree of ocular inflammation at the initial diagnosis, were all risk factors for complications [24]. Previous reports showed that cataract is associated with systemic steroid therapy, the amount of steroid eye drops and degree of ocular inflammation [25-28]. We found that the proportion of patients with complications increased from $31.7 \%$ at the first visit to an ophthalmologist to $56.1 \%$ at the final observation, and it was especially striking that the number of patients with cataract increased 3 -fold over this period. Patients with complications at the initial diagnosis indeed had significantly more complications at the final observation, but there was no association between the presence of complications and age 
at diagnosis of uveitis, or the time from arthritis onset to uveitis diagnosis, and ANA-positivity. There were also no significant differences between complications and persisting active uveitis at the final observation. In addition, we found that systemic steroid therapy had no influence on cataract formation, and the presence of cataract at the first visit had no influence on the surgical history. JIA-U carries a risk of decreased visual acuity and blindness [29, 30]. While none of the patients lost their eyesight over the observation period in our study, more than half did not fully recover visual acuity or had decreased visual acuity at the final observation. Steroid eye drops, oral MTX, and biological agents (IFX and ADA) were mainly used for treatment, and especially biological agents had been employed in a high proportion of patients. This may be one of the reasons why there was no loss of eyesight and few patients with severely decreased vision in our study.

While female sex is reported as a risk factor for JIA-U, severity of the uveitis may be greater in males [31-33]. In comparison, in our study, female sex was not a risk factor for JIA-U, but males with uveitis did have a significantly higher incidence of active uveitis at the last observation.

This study has some limitations. It included 726 Japanese JIA patients, representing approximately $1 / 4$ of all JIA patients in Japan and an estimated half of all patients treated in medical centers specialized in pediatric rheumatic diseases. Although Japan is divided into 47 prefectures, there are only about 80 pediatric rheumatologists and they are unevenly distribution locally, so general pediatricians treat JIA in areas where there are no pediatric rheumatologists. On the other hand, because severe cases are often referred to a pediatric rheumatologist, many JIA-U will be treated by pediatric rheumatologists. Accordingly, the prevalence of uveitis in Japan as established here may actually be even less than $6 \%$ of all JIA patients.

Just as there are few pediatric rheumatologists, so there are also few ophthalmologists who specialize in uveitis in Japan. In addition, although the standardization of uveitis nomenclature (SUN) working group reported criteria to evaluate uveitis [34], few ophthalmologists in Japan use these criteria. Therefore, in the present study, because pediatricians extracted the records written by each ophthalmologist there were some uninterpretable data, especially the location of uveitis. Because of the retrospective nature of this study, there were also some missing data such as data on visual acuity. Although we issued recommendations for ophthalmologic screening intervals for JIA in 2015 [9], because we issued it one year before this study, most patients were not receiving screening according to this recommendation. Hence, only $34 \%$ of patients had regular ophthalmologic screening before uveitis diagnosis, and 34\% had ocular symptoms at the time of uveitis diagnosis in this study. JIA-U is typically asymptomatic and insidious [2], so if patients have regular ophthalmologic examinations, ocular symptoms may be identified less frequently at the time of JIA diagnosis, but more often beforehand.

We did not collect data on the degree of ocular inflammation and the dose of steroids, and so we were unable to analyze potential associations between uveitis and these factors. Also, we did not collect detailed data on dose and timing of drug use for any drugs other than steroids, so we could not analyze the effects of these treatments on uveitis. Thus, because detailed analysis of treatment was difficult in this study, we plan an additional study in cooperation with ophthalmologists in future.

\section{Conclusions}

We investigated whether the prevalence and characteristics of JIA-U in Japan are different from those reported elsewhere. We conclude that the prevalence of JIA-U in Japan is lower than reported in countries with predominantly Caucasian populations. Risk factors for JIA-U were identified as oligoarthritis, early arthritis onset, ANA-positivity, RF-negativity and anti-CCP antibody-negativity. Arthritis onset was significantly earlier in females. Although the most frequent location of uveitis was anterior, males tended to have more affected locations in other the parts of the eye, and the uveitis remission rate was lower than in females. Thus, in males, uveitis may be more severe and harder to cure. Once uveitis had developed, many patients did not fully recover their eyesight. In addition, we found that uveitis may develop before arthritis onset, or under treatment for arthritis and even after cessation of arthritis medication. It is important to recognize these characteristics when caring for Japanese patients with JIA. Patients under 8 years of age and under 7 years of arthritis onset require especially careful specialist ophthalmological monitoring.

\section{Abbreviations}

ADA: Adalimumab; ANA: Antinuclear antibodies; anti-CCP: anti-Cyclic citrullinated peptides; Cl: Confidence interval; GC: Glucocorticoid; HLAB27: Human leucocyte antigen B27; IFX: Infliximab; ILAR: International League of Associations for Rheumatology; JIA: Juvenile idiopathic arthritis; JIAU: Juvenile idiopathic arthritis associated uveitis; MMP-3: Matrix Metalloproteinase-3; MTX: Methotrexate; NSAID: Non-steroidal antiInflammatory drugs; OR: Odds ratio; PRAJ: Pediatric Rheumatology Association of Japan; SUN: Standardization of Uveitis Nomenclature; TCZ: Tocilizumab

\section{Acknowledgments}

We thank the children and parents participating in the study. We thank Dr. Ryuhei Yasuoka, Dr. Toaki Kohagura, Dr. Naoki Abe, Dr. Haruna Nakaseko, Dr. Shinji Kawabe (Department of Immunology and Infectious Diseases, Aichi Children's Health and Medical Center, Obu, Japan), Dr. Takuji Murata, Dr. Yuko Sugita (Department of Pediatrics, Osaka Medical College, Takatsuki, Japan), Dr. Hiroshi Nihira (Department of Pediatrics, Kyoto University Graduate School of Medicine, Kyoto, Japan), Dr. Kanako Mitsunaga, Dr. Akiko 
Yamaide (Department of Allergy and Rheumatology, Chiba Children's Hospital, Chiba, Japan), Dr. Naoki Shimojo, Dr. Yuzaburo Inoue, Dr. Hironori Sato (Department of Pediatrics, Chiba University Graduate School of Medicine, Chiba, Japan), Dr. Shunichiro Takezaki (Department of Pediatrics, Hokkaido University Graduate School of Medicine, Sapporo, Japan), Dr. Fumiko Okazaki (Department of Pediatrics, Yamaguchi University Graduate School of Medicine, Ube, Japan), Dr. Tomo Nozawa, Dr. Asami Oohara, Dr. Ayako Murase and Dr. Tetsuya Tsuchida (Department of Pediatrics, Yokohama City University Graduate School of Medicine, Yokohama, Japan) for collecting the medical records. We thank Dr. Syuji Takei (Department of Pediatrics, Kagoshima University Graduate School of Medicine, Kagoshima, Japan) for analyzing data. We thank Dr. Norihiro Nishimoto (Department of Molecular Regulation for Intractable Disease, Institute of Medical Science, Tokyo Medical University, Tokyo, Japan) and Dr. Toshihiro Matsui (Department of Rheumatology, Clinical Research Center for Allergy and Rheumatology, National Hospital Organization Sagamihara National Hospital, Sagamihara, Japan) for research planning.

\section{Funding}

This work was supported by Health Labour Sciences Research Grant: 201510096A

\section{Availability of data and materials}

The datasets used and/or analyzed during the current study are not publicly available for ethical reasons, as well as privacy reasons, but are available from the corresponding author on reasonable request.

\section{Authors' contributions \\ All authors were involved in the conception, design of the study and revising it critically for important intellectual content. JY and MY were involved in the acquisition of data, analysis, interpretation of data and drafting of the manuscript. All authors read and approved the final manuscript.}

\section{Ethics approval and consent to participate}

This is a retrospective study, approved by the Ethics Review Board of Tokyo Medical and Dental University (No.M2015-537), the main study center, on March 4th, 2016, and then approval was obtained from the 15 additional medical centers. Guardians and patients were provided information by means of an opt-out form.

\section{Consent for publication}

Not applicable.

\section{Competing interests}

Tokyo Medical and Dental University (TMDU) received unrestricted research grants for Department of Lifetime Clinical Immunology from AbbVie GK, Ayumi Pharmaceutical, Chugai Pharmaceutical, CSL Behring, Japan Blood Products Organization, Mitsubishi Tanabe Pharma, Nippon Kayaku, Ono Pharmaceutical, Towa Pharmaceutical, and UCB Japan. TMDU paid the salary of Masaaki Mori.

The authors declare that they have no competing interests.

\section{Publisher's Note}

Springer Nature remains neutral with regard to jurisdictional claims in published maps and institutional affiliations.

\section{Author details}

'Department of Pediatrics, Hiroshima University Graduate School of Biomedical and Health Sciences, 1-2-3 Kasumi, Minami-ku, Hiroshima 734-8551, Japan. ²Department of Pediatrics, Okayama University Hospital, 2-5-1 Shikata-cho, Kita-ku, Okayama 700-8558, Japan. ${ }^{3}$ Department of Pediatrics, Osaka Medical College, 2-7 Daigaku-machi, Takatsuki 569-8686, Japan. ${ }^{4}$ Department of General Pediatrics, Miyagi Children's Hospital, 4-3-17 Ochiai, Aoba-ku, Sendai 989-3126, Japan. ${ }^{5}$ Department of Immunology and Infectious Diseases, Aichi Children's Health and Medical Center, 7-426 Morioka-cho, Obu, Aichi 474-8710, Japan. ${ }^{6}$ Department of Pediatrics, KKR Sapporo Medical Center, 6-3-40 Hiragishi 1-jo, Toyohira-ku, Sapporo 062-0931, Japan. 7 Department of Pediatrics, Kagoshima University Hospital, 8-35-1 Sakuragaoka, Kagoshima 890-0075, Japan. ${ }^{8}$ Department of Pediatrics, Graduate School of Medical Sciences, Kanazawa University, 13-1
Takara-machi, Kanazawa 920-8641, Japan. ${ }^{9}$ Department of Allergy and Rheumatology, Chiba Children's Hospital, 579-1 Heta-cho, Midori-ku, Chiba 266-0007, Japan. ${ }^{10}$ Department of Pediatric Rheumatology, Hyogo Prefectural Kobe Children's Hospital, 1-6-7 Minatojimaminami-machi, Chuo-ku, Kobe 650-0047, Japan. ${ }^{11}$ Department of Pediatrics, Yokohama City University Graduate School of Medicine, 3-9 Fukuura, Kanazawa-ku, Yokohama 236-0004, Japan. ${ }^{12}$ Department of Pediatrics, Kyoto University Graduate School of Medicine, 54 Shogoin Kawahara-cho, Sakyo-ku, Kyoto 606-8507, Japan. ${ }^{13}$ Department of Pediatrics, Chiba University Graduate School of Medicine, 1-8-1 Inohana, Chuo-ku, Chiba 260-8670, Japan. ${ }^{14}$ Department of Pediatrics, Yamaguchi University Graduate School of Medicine, 1-1-1 Minamikogushi, Ube 755-8505, Japan. ${ }^{15}$ Department of Lifetime Clinical Immunology, Graduate School of Medical and Dental Sciences, Tokyo Medical and Dental University, 1-5-45 Yushima, Bunkyo-ku, Tokyo 113-8510, Japan.

Received: 7 January 2019 Accepted: 1 April 2019

Published online: 11 April 2019

\section{References}

1. Saurenmann RK, Levin AV, Feldman BM, Rose JB, Laxer RM, Schneider R, et al. Prevalence, risk factors, and outcome of uveitis in juvenile idiopathic arthritis: a long-term followup study. Arthritis Rheum. 2007;56:647-57.

2. Heiligenhaus A, Niewerth M, Ganser G, Heinz C, Minden K. German uveitis in childhood study group. Prevalence and complications of uveitis in juvenile idiopathic arthritis in a population-based nation-wide study in Germany: suggested modification of the current screening guidelines. Rheumatology. 2007:46:1015-9.

3. Kotaniemi K, Sihto-Kauppi K, Salomaa P, Säilä H, Ristolainen L, Kauppi M. The frequency and outcome of uveitis in patients with newly diagnosed juvenile idiopathic arthritis in two 4-year cohorts from 1990-1993 and 20002003. Clin Exp Rheumatol. 2014;32:143-7.

4. Angeles-Han ST, Pelajo CF, Vogler LB, Rouster-Stevens K, Kennedy C, Ponder $L$, et al. Risk markers of juvenile idiopathic arthritis-associated uveitis in the childhood arthritis and rheumatology research Alliance (CARRA) registry. J Rheumatol. 2013:40:2088-96.

5. Nordal E, Rypdal V, Christoffersen T, Aalto K, Berntson L, Fasth A, et al. Incidence and predictors of uveitis in juvenile idiopathic arthritis in a Nordic long-term cohort study. Pediatr Rheumatol. 2017;15:66.

6. Yu HH, Chen PC, Wang LC, Lee JH, Lin YT, Yang YH, et al. Juvenile idiopathic arthritis-associated uveitis: a Nationwide population-based study in Taiwan. PLoS One. 2013;8:e70625.

7. Kotaniemi K, Savolainen A, Karma A, Aho K. Recent advances in uveitis of juvenile idiopathic arthritis. Surv Ophthalmol. 2003:48:489-502.

8. Berk AT, Koçak N, Ünsal E. Uveitis in juvenile idiopathic arthritis. Ocul Immunol Inflamm. 2001;9:243-51.

9. Okamoto N, Iwata N, Umebayashi H, Okura Y, Kinjo N, Kunishima T, et al. Guidance for early treatment of juvenile idiopathic arthritis. Tokyo: Medical Review Corporation; 2015.

10. Okamoto N, Yokota S, Takei S, Okura Y, Kubota T, Shimizu M, et al. Clinical practice guidance for juvenile idiopathic arthritis (JIA) 2018. Mod Rheumatol. 2018;29:1-19. https://doi.org/10.1080/14397595.2018.1514724 [Epub ahead of print].

11. American Academy of Pediatrics Section on Rheumatology and Section on Ophthalmology: Guidelines for ophthalmologic examinations in children with juvenile rheumatoid arthritis. Pediatrics. 1993;92:295-6.

12. Petty PE, Southwood TR, Manners P, Baum J, Glass DN, Goldenberg J, et al. International league of associations for rheumatology classification of juvenile idiopathic arthritis: second revision, Edmonton, 2001. J Rheumatol. 2004:31:390-2.

13. Ferris FL 3rd, Kassoff A, Bresnick GH, Bailey I. New visual acuity charts for clinical research. Am J Ophthalmol. 1982;94:91-6.

14. Carvounis PE, Herman DC, Cha S, Burke JP. Incidence and outcomes of uveitis in juvenile rheumatoid arthritis, a synthesis of the literature. Graefes Arch Clin Exp Ophthalmol. 2006;244:281-90.

15. Kotaniemi K, Kautiainen H, Karma A, Aho K. Occurrence of uveitis in recently diagnosed juvenile chronic arthritis: a prospective study. Ophthalmology. 2001;108:2071-5.

16. Sim KT, Venning HE, Barrett S, Gregson RM, Amoaku WM. Extended oligoarthritis and other risk factors for developing JIA-associated uveitis under ILAR classification and its implication for current screening guideline. Ocul Immunol Inflamm. 2006;14:353-7. 
17. Petty R, Laxer R, Lindsley C, Wedderburn L. Textbook of pediatric rheumatology, vol. 119. 7th ed. Philadelphia: Elsevier; 2015.

18. Angeles-Han ST, McCracken C, Yeh S, Jenkins K, Stryker D, Rouster-Stevens K, et al. Characteristics of a cohort of children with Juvenile Idiopathic Arthritis and JIA-associated Uveitis. Pediatric Rheumatol. Online J. 2015;13:-19. https://doi.org/10.1186/s12969-015-0018-8.

19. Chen CS, Roberton D, Hammerton ME. Juvenile arthritis-associated uveitis: visual outcomes and prognosis. Can J Ophthalmol. 2004;39:614-20.

20. Nordal EB, Songstad NT, Berntson L, Moen T, Straume B, Rygg M. Biomarkers of chronic uveitis in juvenile idiopathic arthritis: predictive value of antihistone antibodies and antinuclear antibodies. J Rheumatol. 2009;36: $1737-43$.

21. Yu EN, Meniconi ME, Tufail F, Baltatzis S, Foster CS. Outcomes of treatment with immunomodulatory therapy in patients with corticosteroid-resistant juvenile idiopathic arthritis-associated chronic iridocyclitis. Ocul Immunol Inflamm. 2005;13:353-60.

22. Heiligenhaus A, Mingels A, Heinz C, Ganser G. Methotrexate for uveitis associated with juvenile idiopathic arthritis: value and requirement for additional anti-inflammatory medication. Eur J Ophthalmol. 2007;17:743-8.

23. Foeldvari I, Wierk A. Methotrexate is an effective treatment for chronic uveitis associated with juvenile idiopathic arthritis. J Rheumatol. 2005;32: $362-5$.

24. Woreta F, Thorne JE, Jabs DA, Kedhar SR, Dunn JP. Risk factors for ocular complications and poor visual acuity at presentation among patients with uveitis associated with juvenile idiopathic arthritis. Am J Ophthalmol. 2007; 143:647-55.

25. Wolf MD, Lichter PR, Ragsdale CG. Prognostic factors in the uveitis of juvenile rheumatoid arthritis. Ophthalmology. 1987;94:1242-8.

26. Kump LI, Castañeda RA, Androudi SN, Reed GF, Foster CS. Visual outcomes in children with juvenile idiopathic arthritis-associated uveitis. Ophthalmology. 2006;113:1874-7.

27. Angeles-Han S, Yeh S. Prevention and management of cataracts in children with juvenile idiopathic arthritis-associated uveitis. Curr Rheumatol Rep. 2012;14:142-9.

28. Thorne JE, Woreta FA, Dunn JP, Jabs DA. Risk of cataract development among children with juvenile idiopathic arthritis-related uveitis treated with topical corticosteroids. Ophthalmology. 2010;117:1436-41.

29. Thorne JE, Woreta F, Kedhar SR, Dunn JP, Jabs DA. Juvenile idiopathic arthritis-associated uveitis: incidence of ocular complications and visual acuity loss. Am J Ophthalmol. 2007;143:840-6.

30. de Boer J, Wulffraat N, Rothova A. Visual loss in uveitis of childhood. Br J Ophthalmol. 2003;87:879-84.

31. Holland GN, Denove CS, Yu F. Chronic anterior uveitis in children: clinical characteristics and complications. Am J Ophthalmol. 2009;147:667-78.

32. Chia A, Lee V, Graham EM, Edelsten C. Factors related to severe uveitis at diagnosis in children with juvenile idiopathic arthritis in a screening program. Am J Ophthalmol. 2003;135:757-62.

33. Kalinina Ayuso V, Ten Cate HA, van der Does P, Rothova A, de Boer JH. Male gender and poor visual outcome in uveitis associated with juvenile idiopathic arthritis. Am J Ophthalmol. 2010;149:987-93.

34. Jabs DA, Nussenblatt RB, Rosenbaum JT, Standardization of uveitis nomenclature (SUN) working group. Standardization of uveitis nomenclature for reporting clinical data. Results of the first international workshop. Am J Ophthalmol. 2005;140:509-16.

\section{Ready to submit your research? Choose BMC and benefit from:}

- fast, convenient online submission

- thorough peer review by experienced researchers in your field

- rapid publication on acceptance

- support for research data, including large and complex data types

- gold Open Access which fosters wider collaboration and increased citations

- maximum visibility for your research: over $100 \mathrm{M}$ website views per year

At BMC, research is always in progress.

Learn more biomedcentral.com/submissions 